\title{
ACCELERATION OF HYPERFRACTIONATED CHEMORADIATION REGIMEN FOR ADVANCED HEAD AND NECK CANCER
}

\author{
Aaron M. Allen, MD, ${ }^{1 *}$ Mohamed Elshaikh, MD, ${ }^{1}$ Francis P. Worden, $\mathrm{MD},{ }^{2}$ Carol R. Bradford, $\mathrm{MD},{ }^{3}$ \\ Theodoros N. Teknos, MD, ${ }^{3}$ Douglas B. Chepeha, MD, ${ }^{3}$ Christina Tsien, MD, ${ }^{1}$ Laura A. Dawson, MD, ${ }^{1}$ \\ Susan Urba, MD, ${ }^{2}$ Gregory T. Wolf, MD, ${ }^{3}$ Daniel Normolle, PhD, ${ }^{4}$ Avraham Eisbruch, MD ${ }^{1}$ \\ ${ }^{1}$ Department of Radiation Oncology, University of Michigan, Ann Arbor, Michigan. \\ E-mail: eisbruch@umich.edu \\ ${ }^{2}$ Department of Internal Medicine, University of Michigan, Ann Arbor, Michigan \\ ${ }^{3}$ Department of Otolaryngology/Head and Neck Surgery, University of Michigan, Ann Arbor, Michigan \\ ${ }^{4}$ Department of Biostatistics, University of Michigan, Ann Arbor, Michigan
}

Accepted 11 May 2006

Published online 16 November 2006 in Wiley InterScience (www.interscience.wiley.com). DOI: 10.1002/hed.20495

\begin{abstract}
Background. Our aim was to evaluate the acceleration of a hyperfractionated, concurrent chemoradiation regimen (HxCRT) for advanced head and neck squamous cell carcinoma (HNSCC).

Methods. Patients with unresectable HNSCC were treated based on a previously published HxCRT regimen: 1.25 Gy twice daily to 70 Gy concurrent with cisplatin $12 \mathrm{mg} / \mathrm{m}^{2} /$ day and 5-fluorouracil $600 \mathrm{mg} / \mathrm{m}^{2} /$ day for 5 days, weeks 1 , 5. This regimen was accelerated in this series by shortening the treatment from 7 to 6 weeks by omitting the planned mid-treatment 1-week break.

Results. Forty-six patients with T3-4/N3 disease were treated. The main acute toxicity was pharyngeal. Median weight change during therapy in patients with and without enteral feeding tubes was $-3.8 \%$ and $-7.9 \%$, respectively $(p=.08)$. Fifteen percent had late grade III pharyngeal toxicity. Local/regional and distant failure rates were $28 \%$ and $17 \%$, respectively; $52 \%$ are alive without evidence of disease.

Conclusions. In nonresectable HNSCC, acceleration of the $\mathrm{H} \times \mathrm{CRT}$ regimen is feasible, requiring enteral feeding tubes during
\end{abstract}

Correspondence to: A. Eisbruch

${ }^{*}$ Current address: Department of Radiation Oncology, Dana-Farber Cancer Institute, 44 Binney St., Boston Massachusetts 02115.

Contract grant sponsor: Thomas Duke Family Head and Neck Cancer Research Fund.

๑) 2006 Wiley Periodicals, Inc. therapy in most patients. (C) 2006 Wiley Periodicals, Inc. Head Neck 29: 137-142, 2007

Keywords: head and neck cancer; radiotherapy; chemotherapy; altered fractionation; dysphagia

Locally advanced squamous cell carcinoma of the head and neck (HNSCC) remains a challenging disease to treat, with overall survival with current therapy of $50 \%{ }^{1}$ Recent approaches to improve standard treatment have included: adding concurrent chemotherapy to radiation, ${ }^{2,3}$ altered fractionated irradiation, ${ }^{4}$ and combinations of altered fractionation and concurrent chemotherapy. ${ }^{1,5,6}$ In 1998, Brizel et al, ${ }^{1}$ from Duke University, published a randomized study comparing hyperfractionated chemoradiation (HxCRT) to hyperfractionated radiotherapy (RT) alone in locally advanced HNSCC. They demonstrated that the addition of concurrent chemotherapy to hyperfractionated RT resulted in a significant advantage in local tumor control and a borderline significant benefit in overall survival, compared with hyperfractionated RT alone. 
An important limitation of the intensification of the treatment regimens is their accompanying toxicity. Frequent grade III-IV mucositis and significant weight loss have become expected toxicities of aggressive regimens. ${ }^{1,5-7}$ To manage these toxicities, the Duke University HxCRT protocol required a 1-week planned treatment break in mid-therapy. Another strategy that has been used to reduce treatment-related weight loss and to prevent hospitalizations and treatment breaks is prophylactic enteral feeding tubes. ${ }^{8-10}$

We report in this paper our experience in accelerating the HxCRT regimen (AccHxCRT) for patients with locally advanced HNSCC. The patients selected for this series had unresectable local/ regional disease that precluded them from other institutional protocols of organ preservation that were open at the time (in those protocols, resectable disease was an eligibility criterion and induction chemotherapy was used to select patients for surgery vs. chemoradiation). Acceleration was achieved by the elimination of the planned 1-week treatment break in the original HxCRT regimen, resulting in reduced overall treatment time from 7 to 6 weeks, and prophylactic enteral feeding tubes were used in an effort to reduce weight loss during therapy. We expected this regimen modification to be beneficial, in view of local/regional tumor control advantages gained by reduced overall treatment time to a similar extent in randomized studies of accelerated radiation alone. ${ }^{4,11}$

\section{MATERIALS AND METHODS}

Patients and Therapy. This study was approved by the institutional review boards of the University of Michigan and the affiliated Veterans Administration Hospital, and all patients underwent detailed informed consent. Eligible patients included patients without evidence of distant metastatic disease whose tumors were judged to be nonresectable. Nonresectability criteria included neck metastases encircling the carotid artery, tumors involving the prevertebral fascia, and nonnasopharyngeal tumors extending to the base of skull. In addition, patients in whom surgery would have required laryngo-glosso-pharyngectomy and patients with significant nodal involvement of the low neck whose prognosis was judged to be poor and therefore not recommended for extensive surgery were eligible for this treatment protocol. Radiation was given in 1.25-Gy fractions twice a day at least 6 hours apart. A total dose of 70 Gy was delivered to the gross tumor volume over 6 weeks without any planned treatment breaks. Target volumes at risk of subclinical disease received 50 to 60 Gy. Conformal 3-dimensional radiotherapy techniques were used, including beam's-eye views to ensure adequate target coverage. The spinal cord was shielded at $40 \mathrm{~Gy}$. Dose homogeneity was restricted to $\pm 7 \%$ prescribed dose. No specific effort was made to spare the salivary glands in most of these patients because of clinic-related constraints on lengthy treatment time in patients receiving twice-daily therapy. Optic nerve doses were limited to maximum of 54 Gy. No reduced dose was required for any substantial part of the gross disease in any of the 8 patients with paranasal sinus cancer. Chemotherapy consisted of 5 -fluorouracil (5-FU) given as continuous infusion, $600 \mathrm{mg} / \mathrm{m}^{2} /$ day, and cisplatin, $12 \mathrm{mg} / \mathrm{m}^{2} /$ day, for 5 days in weeks 1 and 5 . Hydration and antiemetics during chemotherapy were delivered according to standard clinical care.

All patients received narcotics, mostly starting in the third week and tapered-off on average at 1 month after completing RT. Prophylactic nystatin was provided routinely, and patients developing overt fungal infection received fluconazole. Twice-weekly intravenous fluids were delivered to patients with $>10 \%$ weight loss (besides cisplatinrelated hydration). A dedicated team consisted of a nurse, nutritionist, and physician team during treatment. Patients with dysphagia more than 3 months after therapy were referred to the Speech and Swallowing Therapy service for evaluation.

All patients in whom the pharynx was included in the high-dose treatment volumes (patients with oropharyngeal, hypopharyngeal, and laryngeal primaries, or large neck nodes) were referred for percutaneous endoscopic gastrostomy (PEG) at the start of treatment in order to improve treatment tolerance. Patients were assessed weekly and following treatment every 6 to 8 weeks during the first 2 years and every 3 months thereafter for acute and late toxicity. Progression was defined primarily by clinical exam and contrast-enhanced CT scans performed at 6-month intervals. Toxicity was scored using the Radiation Therapy and Oncology Group (RTOG) Acute and Late Radiation Morbidity Scoring Criteria. In addition, patients had weekly recording of weight while on treatment.

Survival was calculated from the date of treatment initiation to the date of death or last followup. Survival curves were calculated by the productlimit (Kaplan-Meier) method. Confidence intervals 
for binomial probabilities were calculated by the likelihood ratio method. Two-tailed tests are reported. Local-regional failure was reported by cumulative incidence, ${ }^{12}$ which allows estimation of incidence rates in the presence of competing risks from other causes. Statistical calculations were performed using SAS version 9.1.

\section{RESULTS}

Forty-six patients with HNSCC were treated with AccHxCRT between November 1999 and December 2003. Patient characteristics are displayed in Table 1. Ninety-one percent of the patients had American Joint Committee on Cancer (AJCC) stage IV disease, with the remainder having stage III disease; all had tumor (T) classification 3-4 except for 1 patient with an unknown primary and advanced neck disease. Fifty-nine percent had oropharyngeal cancers, with base of tongue the most common subsite. Other prominent sites included paranasal sinuses $(13 \%)$ and oral cavity $(9 \%)$.

The treatment course was completed in almost all patients within 6 weeks. Three patients had each a treatment break of more than 3 days due to toxicity or poor compliance. Median delivered RT dose was 70 Gy. All but 2 patients received 70 Gy, with the exception of 1 patient who refused the final treatment and received 67.5 Gy and 1 patient who received a small-volume boost to total 78.5 Gy. Full dose chemotherapy was received by $36 / 46$ $(78 \%)$ patients, with the remaining 10 patients completing only a single cycle or requiring chemo-

Table 1. Patient and tumor characteristics.

\begin{tabular}{lc}
\hline Characteristic & No. of patients \\
\hline Sex & \\
Males & 36 \\
Females & 10 \\
Race & \\
Caucasians & 43 \\
African-Americans & 3 \\
Median age (range), y & $56(31-78)$ \\
Tumor site & \\
Oropharynx & 27 \\
Paranasal sinuses/nasal cavity & 8 \\
Oral cavity & 4 \\
Larynx & 3 \\
Hypopharynx & 2 \\
Unknown primary/other & 2 \\
Tumor stage & \\
T3-4, N0-1 & 16 \\
T3-4, N2 & 24 \\
T3-4, N3 & 5 \\
TxN3 & 1 \\
\hline
\end{tabular}

Intensive Chemoradiation in Head and Neck Cancer
Table 2. Common acute and late severe toxicities. ${ }^{*}$

\begin{tabular}{lc}
\hline Toxicity & No. of patients \\
\hline Acute toxicity & \\
Mucositis & 21 \\
$\quad$ Grade II & 24 \\
Grade III & \\
Pharyngeal & 5 \\
$\quad$ Grade II & 37 \\
Grade III & \\
Skin desquamation & 21 \\
$\quad$ Grade II & 6 \\
Grade III & \\
Late toxicity & 7 \\
Pharyngeal/esophageal & 7 \\
$\quad$ Grade II & 1 \\
$\quad$ Grade III & 1 \\
Osteoradionecrosis & 1 \\
Skin necrosis requiring surgery & 1 \\
Nasal stenosis & \\
Hearing loss & \\
\hline *Grade V toxicities included 1 each with neutropenic sepsis and aspi- \\
ration pneumonia, and 1 cerebrovascular event (possibly treatment \\
related).
\end{tabular}

therapy dose reductions in the second cycle, due to severe mucositis/skin desquamation, or due to patient refusal to receive a second cycle.

Forty-two of $46(91 \%)$ patients had their oral cavity, oropharynx, or hypopharynx in the highdose RT volumes (excluding 4 patients with paranasal cancer). Twenty-two (52\%) of these patients had prophylactic PEG placement. The reasons for nonplacement were patient refusal or need to start therapy before a PEG could be placed. Of the patients without prophylactic PEG, 9 received PEG during treatment and 5 had PEG within 3 weeks after the completion of treatment for progressive dysphagia and weight loss.

Severe toxicity data is summarized in Table 2. Three patients $(6.5 \%)$ died of treatment-related (neutropenic sepsis and aspiration pneumonia) or possible treatment-related (cerebrovascular event 4 months after therapy) causes. Mucositis and dysphagia were the most prevalent severe acute toxicities. The median weight change relative to pretherapy in all patients was $-6.2 \%$ (range, $-32.9 \%$ to $+11.2 \%$ ). The median percentage weight change in patients without prophylactic feeding tube was $-7.9 \%$ (range, $-32.9 \%$ to $+7.8 \%$ ), compared with median weight change of $-3.8 \%$ (range, $-19.2 \%$ to $+11.2 \%$ ) in patients with prophylactic feeding tube $(p=.08)$. Moist skin desquamation was observed in six $(13 \%)$ patients.

Late grade II-III toxicity excluding xerostomia was observed in $18 / 46(39 \%)$ patients, and the 
most common was dysphagia. Fourteen patients required PEG for $>3$ months for ongoing nutritional support, and 5 patients required PEG support beyond 6 months, with 1 patient requiring PEG for 2 years. Strictures requiring dilation occurred in 7 patients. All were detected during the workup of dysphagia or aspiration between 2 and 8 months after the completion of therapy. The workup in all these patients included videofluoroscopy and laryngoscopy/esophagoscopy under anesthesia. The primary tumor sites in patients with strictures were posterior pharyngeal wall (3 patients), oropharynx (3 patients), and hypopharynx ( 1 patient). In $6 / 7$ patients, the strictures were located in the post-cricoid hypopharynx, and in 1 patient in the upper esophagus. The doses delivered to the sites of strictures were $70 \mathrm{~Gy}(4 \mathrm{pa}-$ tients), 60 Gy (1 patient), and 50 Gy (2 patients). Following dilation of the strictures, 3 patients continued to depend on tube feeding, 2 patients ate most of their food but required supplemental tube feeding, and 2 patients did not require tube feeding.

At a median follow-up of 31 months (range, 669 months), 17 patients (37\%) have had documented clinical failure, 15 of whom died of disease. Thirteen patients ( $28 \%$ ) experienced local/regional failure (see Figure 1), 4 of whom also had distant failure, and 4 patients had distant failure alone. All local failures were in field. Four patients died of other causes; 3 were related to toxicity, or possi-

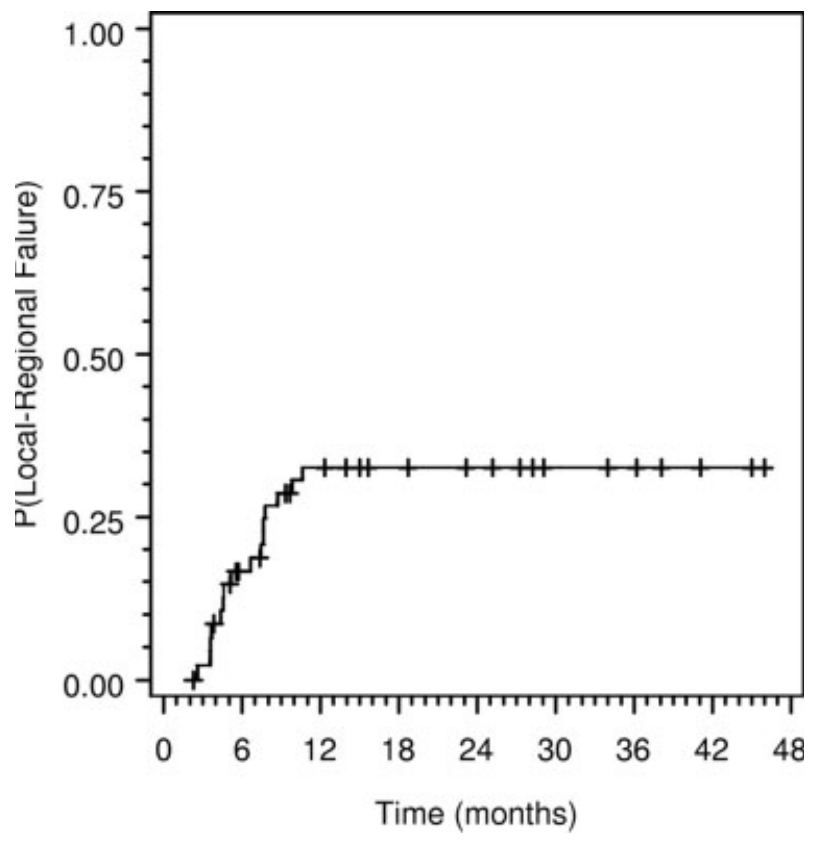

FIGURE 1. Locoregional failures.

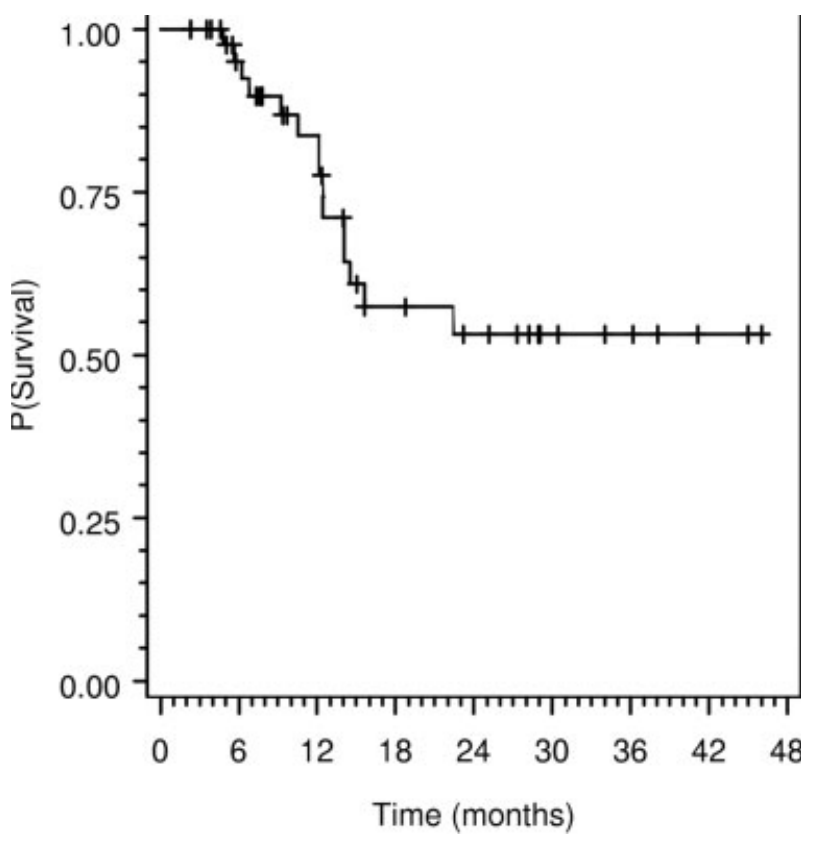

FIGURE 2. Actuarial overall survival.

ble toxicity, as detailed above, and 1 patient died of lung cancer. One patient was lost to follow-up. Twenty-four patients $(52 \%)$ remain alive without evidence of disease (Figures 2 and 3 ).

\section{DISCUSSION}

This study demonstrates that an acceleration of the HxCRT regimen, enacted by removing the 1-week treatment break from the original protocol, is feasible when accompanied by temporary enteral feeding in most patients. This conclusion is limited by lack of compliance of many patients with the original recommendation to have feeding tubes inserted prior to therapy.

The importance of weight loss as a predictor of poor outcome has been documented in many cancers, including lung, esophageal, and head and neck cancers. ${ }^{13-15}$ Weight loss during chemoradiation of head and neck cancer has also been found to be correlated with cisplatin-related nephrotoxicity. ${ }^{16}$ The best approach to maintaining weight and minimizing weight loss during treatment of HNSCC with RT has been shown in multiple studies to be PEG placement. ${ }^{9,10,17}$ PEG appears to be superior to jejunal feeding tubes. ${ }^{18}$ However, it is possible that PEG may increase the risk of strictures compared with nasogastric feeding tubes. ${ }^{18}$ This issue has been controversial. ${ }^{19}$

In the current study we have observed a marginally statistically significant difference in weight 


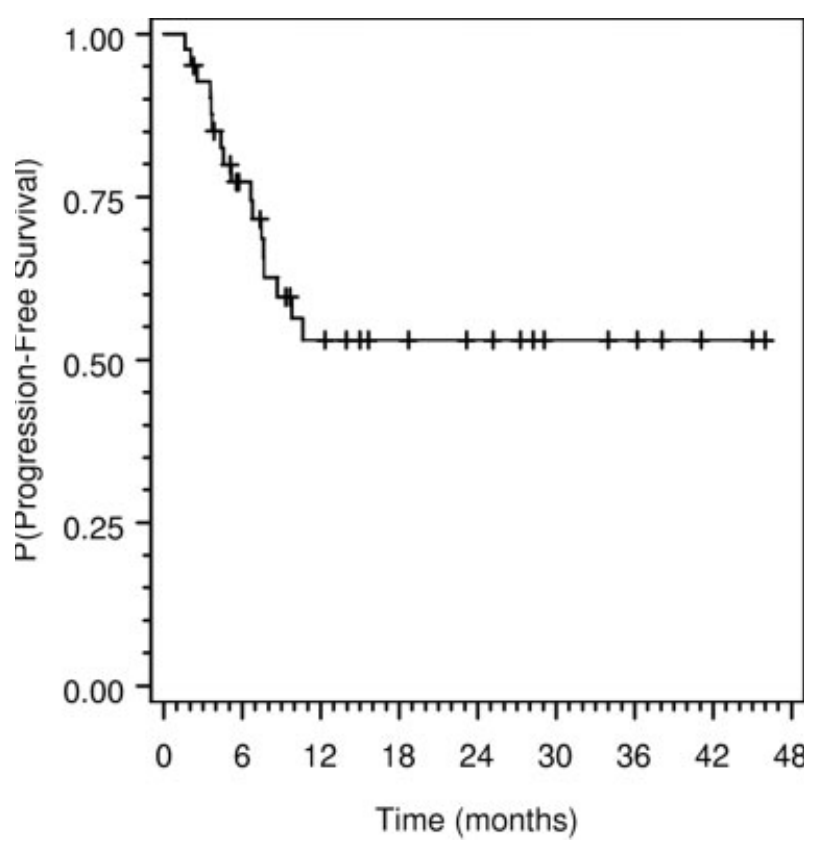

FIGURE 3. Actuarial progression-free survival.

loss during treatment among those patients who had PEG placed compared to those who did not. The weight loss in patients with prophylactic PEG compares favorably to the average $10 \%$ weight loss seen in the Duke randomized trial, in which only $44 \%$ of patients received PEG, only few of which placed before therapy. ${ }^{1}$ Our results mirror the effect of PEG on weight loss reported in a study of patients treated at the University of Pennsylvania with either chemotherapy and standard fractionation RT or hyperfractionated therapy. ${ }^{8}$ Two additional studies by Adelstein et $\mathrm{al}^{2,5}$ also confirm the importance of PEG placement in the maintenance of weight during treatment.

An important factor in predicting outcome of HNSCC is the amount and duration of required treatment breaks. A number of studies have shown that radiation treatment breaks of a week or more are correlated with a poor outcome. ${ }^{20,21}$ However, these studies were conducted in patients whose treatment breaks were caused by acute toxicity, who might have had adverse prognostic features. The effect of a planned treatment break, as done in the original Brizel study, is not as clear. While reducing overall treatment time from 7 to 6 weeks has been found to be beneficial in randomized studies of radiation alone, ${ }^{4,11}$ it is not yet known whether a similar benefit in accelerating radiotherapy is gained when concurrent chemotherapy is added. This issue is being addressed in current randomized studies such as a study by the RTOG of accelerated versus standard fractionated RT, both delivered concurrent with chemotherapy. The result of this and other similar studies will help resolve this issue in the near future. Furthermore, it should be stated that preventing weight loss to eliminate the need for treatment breaks may not result in the same outcome as in unsupported patients who do not lose weight.

The local/regional tumor control and survival rates in our study are comparable to the data from the original HxCRT study of Brizel et al, ${ }^{1}$ with approximately $50 \%$ long-term survival and $70 \%$ local/regional control rate. However, the patients in our study, selected due to their ineligibility for other organ preservation protocols offered at our institution to those with resectable disease, may have had more advanced local/regional disease. Assessing whether or not the elimination of the planned treatment break has resulted in improved local/regional control rates compared with the original protocol, or compared with other trials, would require a study of this regimen in unselected patients with stage III-IV disease.

The main concern in this accelerated regimen is whether the rate of late complications has been excessive. The primary late complication in our study was hypopharyngeal/esophageal strictures and late dysphagia. Strictures requiring dilation occurred in $15 \%$ of the patients. This is equal to the stricture rate of $14 \%$ reported in a study of HxCRT regimen identical to the original Duke protocol, containing a planned treatment break of 5 to 11 days, which was conducted in community hospitals. ${ }^{7}$ A stricture rate of $21 \%$ was reported by investigators from the Cleveland Clinic following $\mathrm{RT}$ concurrent with cisplatin and 5-FU, and twice-daily $\mathrm{RT}$ resulted in a higher rate compared with once-daily $\mathrm{RT}^{22}$ In comparison, lower rates of strictures have been reported following less intensive regimens. Laurell et $\mathrm{al}^{23}$ reported 22 cases of upper esophageal strictures out of $642(3.4 \%)$ patients with head and neck cancer treated with standard fractionated RT alone. In this study, the lowest dose to the sites of strictures was $60 \mathrm{~Gy}$. In comparison, lower doses to the hypopharynx (50 Gy) associated with strictures were found in the current study and following another regimen of intensive chemo-RT. ${ }^{23}$ Thus, concurrent chemo-RT seems to shift the dose-response curve of this complication to the left. However, it does not seem that omitting the mid-treatment break has substantially increased this risk compared with the original, less accelerated regimen. It should be noted that a longer follow-up interval may result in addi- 
tional cases of late dysphagia in our series, whose current median follow-up interval is 2.5 years.

Recent studies of potential strategies to reduce late dysphagia following intensive chemoradiation include the use of radiation protectors ${ }^{24}$ and of intensity-modulated radiotherapy. ${ }^{25}$ The clinical efficacy of these strategies requires validation.

In conclusion, the acceleration of the HxCRT regimen for patients selected due to having advanced nonresectable local/regional disease resulted in tumor control rates similar to those reported for patients who were not selected according to these criteria and had received the original HxCRT regimen. Comparison of the efficacy of the AccHxCRT with other intensive regimens requires its evaluation in nonselected patients with stage III-IV tumors. An aggressive policy of weight management such as prophylactic PEGs is one of the avenues to facilitate the intensification of chemo- RT. Further steps are required in order to reduce late dysphagia in patients treated with similar regimens.

\section{REFERENCES}

1. Brizel DM, Albers ME, Fisher SR, et al. Hyperfractionated irradiation with or without concurrent chemotherapy for locally advanced head and neck cancer. N Engl J Med 1998;338:1798-1804.

2. Adelstein DJ, Li Y, Adams GL, et al. An intergroup phase III comparison of standard radiation therapy and two schedules of concurrent chemoradiotherapy in patients with unresectable squamous cell head and neck cancer. J Clin Oncol 2003;21:92-98.

3. Forastiere AA, Goepfert H, Maor M, et al. Concurrent chemotherapy and radiotherapy for organ preservation in advanced laryngeal cancer. N Engl J Med 2003;349: 2091-2098.

4. Fu KK, Pajak TF, Trotti A, et al. A Radiation Therapy Oncology Group (RTOG) phase III randomized study to compare hyperfractionation and two variants of accelerated fractionation to standard fractionation radiotherapy for head and neck squamous cell carcinomas: first report of RTOG 9003. Int J Radiat Oncol Biol Phys 2000;48:7-16.

5. Adelstein DJ, Saxton JP, Lavertu P, et al. Maximizing local control and organ preservation in stage IV squamous cell head and neck cancer with hyperfractionated radiation and concurrent chemotherapy. J Clin Oncol 2002;20:1405-1410.

6. Ang KK, Harris J, Garden AS, et al. Concomitant boost radiation plus concurrent cisplatin for advanced head and neck carcinomas: radiation therapy oncology group phase II trial 99-14. J Clin Oncol 2005;23:3008-3015.

7. Maguire PD, Meyerson MB, Neal CR, et al. Toxic cure: hyperfractionated radiotherapy with concurrent cisplatin and fluorouracil for stage III and IVA head-and-neck cancer in the community. Int J Radiat Oncol Biol Phys 2004;58:698-704.

8. Lee JH, Machtay M, Unger LD, et al. Prophylactic gastrostomy tubes in patients undergoing intensive irradiation for cancer of the head and neck. Arch Otolaryngol Head Neck Surg 1998;124:871-875.
9. Scolapio JS, Spangler PR, Romano MM, McLaughlin, MP, Salassa, JR. Prophylactic placement of gastrostomy feeding tubes before radiotherapy in patients with head and neck cancer: is it worthwhile? J Clin Gastroenterol 2001;33:215-217.

10. Beaver ME, Matheny KE, Roberts DB, Myers JN. Predictors of weight loss during radiation therapy. Otolaryngol Head Neck Surg 2001;125:645-648.

11. Overgaard J, Hansen HS, Specht L, et al. Five compared with six fractions per week of conventional radiotherapy of squamous-cell carcinoma of head and neck: DAHANCA 6 and 7 randomised controlled trial. Lancet 2003;362:933940 .

12. Kalbfleish JPR. The statistical analysis of failure time data. New York: Wiley; 1980.

13. de Wet M, Falkson G, Rapoport BL. Small cell lung cancer: analysis of factors influencing the response to treatment and survival. Oncology 1994;51:523-534.

14. Feinstein AR, Wells CK. A clinical-severity staging system for patients with lung cancer. Medicine (Baltimore) 1990;69:1-33.

15. Brookes GB. Nutritional status--a prognostic indicator in head and neck cancer. Otolaryngol Head Neck Surg 1985;93:69-74.

16. Lin A, Jabbari S, Worden FP, Dawson LA, Ship JA, Eisbruch A. Metabolic abnormalities associated with weight loss during chemoirradiation of head-and-neck cancer. Int J Radiat Oncol Biol Phys 2005;63:1413-1418.

17. Tyldesley S, Sheehan F, Munk P, et al. The use of radiologically placed gastrostomy tubes in head and neck cancer patients receiving radiotherapy. Int $\mathrm{J}$ Radiat Oncol Biol Phys 1996;36:1205-1209.

18. Mekhail TM, Adelstein DJ, Rybicki LA, Larto MA, Saxton JP, Lavertu P. Enteral nutrition during the treatment of head and neck carcinoma: is a percutaneous endoscopic gastrostomy tube preferable to a nasogastric tube? Cancer 2001;91:1785-1790.

19. Al-Otman M, Amdur R, Morris C, et al. Does feeding tube placement predict for long-term swallowing disability after radiotherapy for head and neck cancer? Head Neck 2003;25:741-747.

20. Suntharalingam M, Haas ML, Van Echo DA, et al. Predictors of response and survival after concurrent chemotherapy and radiation for locally advanced squamous cell carcinomas of the head and neck. Cancer 2001;91:548-554.

21. Herrmann T, Jakubek A, Trott KR. The importance of the timing of a gap in radiotherapy of squamous cell carcinomas of the head and neck. Strahlenther Onkol 1994; 170:545-549.

22. Lee WT, Akst LM, Adelstein DL, et al. Risk factors for hypopharyngeal/upper esophageal strictures formation after concurrent chemoradiation, in Proceedings of the 6th International Conference on Head and Neck Cancer, Washington DC, August 7-11, 2004:s090.

23. Laurell G, Kraepelien T, Mavroidis P, et al. Stricture of the proximal esophagus in head and neck carcinoma patients after radiotherapy. Cancer 2003;97:1693-1700.

24. Haddad R, Wirth L, Costello R, Weeks L, Posner M. Phase II randomized study of concomitant chemoradiation using weekly carboplatin/paclitaxel with or without daily subcutaneous amifostine in patients with newly diagnosed locally advanced squamous cell carcinoma of the head and neck. Semin Oncol 2003;30:84-88.

25. Eisbruch A, Schwartz M, Rasch C, et al. Dysphagia and aspiration after chemoradiotherapy for head-and-neck cancer: which anatomic structures are affected and can they be spared by IMRT? Int J Radiat Oncol Biol Phys 2004;60:1425-1439. 